\title{
Commitment in First-Price Auctions
}

\author{
Yunjian $\mathrm{Xu}^{1(凶)}$ and Katrina Ligett ${ }^{2}$ \\ 1 Engineering Systems and Design, Singapore University of Technology and Design, \\ Singapore, Singapore \\ xuyunijian@gmail.com \\ 2 Computing and Mathematical Sciences and Division of the Humanities and Social \\ Sciences, California Institute of Technology, Pasadena, USA
}

We study a variation of the single-item sealed-bid first-price auction where one bidder (the leader) is given the option to publicly pre-commit to a distribution from which her bid will be drawn. We formulate the auction as a two-stage Stackelberg game: in the first round, one bidder (the leader) is allowed to publicly commit to a mixed strategy; in the second round, the other bidders submit their bids simultaneously. Given the publicly known commitment of the leader, the other bidders simultaneously play (possibly randomized) actions in the second stage.

Intuitively, the leader may have incentive to commit to a distribution with support below what she would have played in a simultaneous first price auction, because in doing so, she induces her opponents to lower their bids as well. Our results provide support for this intuition. For example, we see that even a very simple form of commitment, in which the leader announces to bid zero (effectively, to exit the auction) with some positive probability $p$ and to bid some other announced real number with probability $1-p$, can strictly (and significantly) benefit both bidders in a simple two-bidder setting.

We also completely characterize the optimal commitment strategy for the leader in terms of the bidder valuations, for arbitrary numbers of bidders. The characterized optimal commitment, together with best responses on the part of the followers, forms a subgame perfect equilibrium (SPE). We find that if the leader has the highest or the second highest valuation, then the two bidders with highest valuations strictly benefit from the presence of a committing bidder. This result establishes the leader's optimal commitment as a coordinative mechanism that allows the top two valued bidders to collude without money transfer. Somewhat surprisingly, compared with the simultaneous first-price auction, the leader's optimal commitment yields the top two bidders the same net utility benefit. This observation could eliminate possible conflicts on who should commit and who should follow.

A leader's optimal commitment may result in inefficient outcomes, i.e., the highest-valued bidder does not always win the item. Because the leader's optimal commitment benefits the bidders but hurts the welfare, it must decrease the auctioneer's revenue. Indeed, the auctioneer's revenue strictly decreases whenever the leader (and the highest valued follower) strictly benefits from the commitment.

$\mathrm{Y} . \mathrm{Xu}$-This research was supported in part by NSF grants CCF-0910940 and CNS1254169, the Charles Lee Powell Foundation, and a Microsoft Research Faculty Fellowship. 